\title{
TENDÊNCIAS TEÓRICAS DO GERENCIAMENTO DE RISCOS EM COMPRAS PÚBLICAS SUSTENTÁVEIS: UMA ANÁLISE BIBLIOMÉTRICA A PARTIR DAS BASES SCOPUS E WEB OF SCIENCE
}

Andre Luiz Trajano dos Santos ${ }^{1}$ Augusto da Cunha Reis ${ }^{2}$

\section{RESUMO}

O Gerenciamento de Riscos (GR) tem se tornado um instrumento de mitigação de diversas variá veis que afetam as compras governamentais no curso de seu processo de uma aquisição sustentável. Este artigo tem por objetivo apontar tendências teóricas a partir de um estudo bibliométrico dos principais trabalhos sobre Gerenciamento de Riscos em Compras Públicas Sustentáveis (GRCPS) quanto ao número de publicações por ano, quantidade de citações por autor e países que mais tratam dessa temática, a lém de identificar os autores mais relevantes no tema pesquisado. Para tal, as buscas foram realizadas $n$ a $\mathrm{s}$ bas es científicas Scopus e Web of Science, contemplando os anos de 2010 a 2020, identificando-se ao todo 89 trabalhos para análise bibliométrica por meio do pacote bibliometrix do Software R Studio. Assim, este trabalho tem sua relevância em contribuir com a análise da evolução dos estudos de gerenciamento de riscos em compras públicas sustentáveis ainda incipiente na literatura, uma vez que a pesquisa bibliográfica revela que existem poucos trabalhos no campo acadêmico que aplicam o conceito de gerenciamento de riscos às compras públicas. Este levantamento revela que o interesse na gestão de riscos em compras pública s teve um crescimento de $540 \%$ entre os anos de 2010 e 2020 . Contudo, uma pesquisa futura poderá analisar qualitativamente no portfólio bibliográfico internacional, com a finalidade de se revisar sistematicamente quais categorias de riscos mais afetam a cadeia de suprimentos em razão da a doção de critérios de sustentabilida de em compras públicas.

Palavras-chave: Bibliometria. Cadeia de suprimentos; Compras públicas sustentáveis. Gerenciamento de riscos.

\section{THEORETICAL TRENDS IN RISK MANAGEMENT IN SUSTAINABLE PUBLIC PROCUREMENT: A BIBLIOMETRIC ANALYSIS BASED ON THE SCOPUS AND WEB OF SCIENCE DATABASES}

\begin{abstract}
Risk Management (RM) has become an instrument to mitigate several variables that affect government procurement in the course of its sustainable procurement process. This article aims to point out theoretical trends from a bibliometric study of the main works on Risk Management in Sustainable Public Procurement (RMSPP) regarding the number of publications per year, number of citations per author and countries that deal with this theme the most, in addition to identify the most relevant authors in the researched topic. To this end, searches were carried out in the scientific databases Scopus and Web of Science covering the years 2010 to 2020 , identifying a total of 89 works for bibliometric analysis through the bibliometrix package of the Software R Studio. Thus, this work has its relevance in contributing to the analysis of the evolution of studies on risk management in sustainable public procurement, which is still in its infancy in the literature, since the bibliographical research reveals that there are few works in the academic field that apply the concept of management. risks to public procurement. This survey reveals that interest in risk management in public procurement grew by 540\% between the years 2010 and 2020. However, future research may qualitatively analyze the international bibliograph ic portfolio, in order to systematically review which risk categories most affect the supply chain due to the adoption of sustainability criteria in public procurement.
\end{abstract}

Key words: Bibliometrics. Risk management; Supply chain; Sustainable public procurement.

\footnotetext{
${ }^{1}$ Doutorando do Programa de Pós-Graduação Strict Sen so em Engenharia de Produção e Sistemas do Centro Federal de Ed uc ação Tecnológica Celso Suckow da Fonseca, CEFET/RJ, Rio de Janeiro (Brasil). http://orcid.org/0000-0002-6800-3051 Email: eltrajano@gmail.com

${ }^{2}$ Profes sor do Programa de Pós-Graduação Strict Senso em Engenharia de Produçãoe Sistemas do Centro Fe d eral de Educ ação Tecnológica Celso Suckow da Fonseca, CEFET/RJ, Rio de Janeiro (Brasil). http://orcid.org/0000-0002-3200-8096 Email: augusto.reis@cefet-rj.br
} 


\section{INTRODUÇÃO}

A pesquisa bibliográfica revela que ainda existem poucos artigos que aplicam conceitos de gerenciamento de riscos em compras públicas sustentáveis, visto que o tema de gestão de riscos em compras públicas é um campo de estudo ainda em consolidação (Mogre et al., 2017). O estado, por sua natureza, tem uma grande capacidade de compra, sendo capaz de influenciar uma parte considerável do mercado de bens e de serviços (Cruz et al., 2020). As compras públicas representam cerca de $12 \%$ do produto interno bruto (PIB) dos países membros da Organização para a Cooperação e Desenvolvimento Econômico (Ocde, 2020). De acordo com o Ipea, Instituto de Pesquisa Aplicada, as compras governamentais no Brasil equivalem a uma média de 12,5\% do PIB (IPEA, 2019). Uma cifra considerável a qual não está livre de possíveis riscos, tais como, erro processual na descrição correta do item, falha na entrega, corrupção, quarteis, etc. e mitigar esses riscos é um desafio para o gestor público que objetiva realizar a melhor comprar (Henrique et al., 2020; Yazdani et al., 2019; Aghajanian, 2018; Cortis et al., 2018; Hyari, 2017; Mysiak \& Dionisio PérezBlanco, 2016). Assim, o papel das compras públicas no desenvolvimento sustentável emerge de uma grande discussão nos últimos anos devido à sua participação nos gastos públicos (Zaidi et al., 2019; Brammer \& Walker, 2011).

Os procedimentos de compras ou aquisição pública dos países, em regra, seguem as especificações estabelecidas em seus regulamentos e normativos legais (Bakir et al., 2018; Walker \& Brammer, 2009). É por meio dele que as "regras do jogo" são postas àqueles que pretendem fornecer bens ou serviços para que o estado cumpra o seu papel na prestação de serviços à população. No Brasil, essa regra é regida pela Lei $\mathrm{n}^{\circ} 8.666 \mathrm{de}$ 1993, e, mais recentemente pela Lei $\mathrm{n}^{\circ} 14.133$ de 2021, além de uma série de normativos infralegais os quais complementam todo o arcabouço legal em compras públicas. Tais compras se referem à aquisição de bens e serviços pelo setor público, com vistas a garantir as operações da administração pública e prestar serviços à sociedade, tais como educação, saúde, segurança, energia e infraestrutura (Kashap, 2004; Santos, 2017). Nesse contexto, o gerenciamento das etapas do processo logístico público, como forma de consolidação de seguimentos de mercados, tem se mostrado uma estratégia relevante frente ao alto valor que o estad o é capaz de influenciar. Assim, o gerenciamento de riscos surge como uma questão importante no gerenciamento de qualquer cadeia de suprimentos, ainda mais quando os recursos gastos são públicos (Cruz et al., 2020; Mogre et al., 2017; Aguas et al., 2013).

Por natureza, são nas organizações públicas que bens e serviços são transformados em atendimento aos usuários dos serviços públicos. Diante disso, o gerenciamento de riscos em compras públicas, em face das cadeias de suprimentos por ela influenciadas, se faz necessário tendo em vista que o funcionamento eficaz dessa cadeia, por vezes, depende da mitigação de riscos capazes de alterar o fluxo normal de toda uma rede. Assim, é de vital importância se antecipar a eventos que afetam negativamente o cumprimento da missão de qualquer organização que, porventura, venha participar da cadeia de suprimentos das organizações públicas. Com isso, ao longo dos anos, as melhorias feitas nas operações da cadeia de suprimentos se concentraram principalmente em maneiras de reduzir custos, obter eficiências em escala e mitigação de riscos que por si só é um desafio na administração pública acostumada a gerir volumosos recursos (Wibowo \& Sundermeier, 2020; Aghajanian, 2018; Eckerd \& Girth, 2017; Mehralian et al., 2012; Chan et al., 2011).

Assim, a partir da questão norteadora de pesquisa, visando identificar quais são os autores, artigos e periódicos mais relevantes sobre o gerenciamento de riscos em 
compras públicas sustentáveis na última década, esse trabalho de pesquisa tem por objetivo apontar tendências teóricas a partir de levantamento bibliográfico nas bases Scopus e Web of Science publicados entre os anos de 2010 e 2020, por meio de um estudo bibliométrico dos principais trabalhos sobre o gerenciamento de riscos em compras públicas sustentáveis.

Cabe destacar que bibliometria é o recurso utilizado como um método de análise quantitativa que mensura a contribuição do conhecimento científico a partir das publicações em determinadas áreas do conhecimento (Derviş, 2019; Matos et al., 2017). Logo, buscou-se conhecer o número de publicações por ano, a quantidade de citações por autor e países que mais tratam dessa temática, além de identificar os autores mais relevantes no tema pesquisado bem como da convergência temática das palavras-chave das pesquisas realizadas.

Para tal, esse trabalho está estruturado em cinco capítulos, sendo o primeiro composto por uma breve introdução, seguido pelo referencial teórico. No terceiro, são apresentados os aspectos metodológicos seguindo pelo capítulo de análise dos dados coletados, e por fim as conclusões a partir das análises bibliométricas realizadas.

\section{REFERENCIAL TEÓRICO}

Apresenta-se, nesta seção, o referencial teórico da convergência temática pesquisada a qual, inicialmente faz-se uma incursão teórica do gerenciamento de riscos em compras públicas e as práticas de sustentabilidade em compras públicas, discorrendo sobre o papel do estado como promotor de critérios de sustentabilidade nas aquisições e contrações públicas.

\subsection{Gerenciamento de riscos em compras públicas (GRCP)}

As pesquisas sobre gerenciamento de riscos tiveram um considerável crescimento na última década em razão da busca por melhorar os custos do investimento em ações logísticas em diversos setores. Contudo, o gerenciamento de riscos em compras públicas ainda é pouco estudado pelos pesquisadores do tema, menos ainda quando associado a critérios de desenvolvimento sustentável (Senna et al., 2020; Chiarini et al., 2017; Eckerd \& Girth, 2017). Os riscos estão geralmente atrelados às oportunidades que o negócio vislumbra em seu segmento de atuação, os quais podem ser entendidos como a probabilidade ou possibilidade de um infortúnio, insucesso assim como um resultado indesejado em face de um objetivo a ser alcançado (Kalvet \& Lember, 2010; Alexandre et al., 2004). Por exemplo, no serviço público, o risco da não entrega de um produto ou serviço contratado pode significar a ineficiência da prestação de um determinado serviço à população, assim como o desconhecimento das normas de compras públicas pode significar o limiar entre uma boa ou má aquisição, acarretando aumento em custos logísticos e operacionais.

Em se tratando de compras públicas, a gestão de riscos tem ganhado seu espaço, já que o estado, como responsável em influenciar uma fatia relevante do mercado, deve se antever a falhas que afetam seus processos logísticos seja qual for o seguimento organizacional. Logo, o gerenciamento de riscos deve se concentrar na compreensão dos riscos e na minimização de seu impacto (Senna et al., 2020; Oliveira et al., 2016; Norrman \& Jansson, 2004). Com isso, o gerenciamento de riscos deve cuidar da identificação, análise e do controle ou acompanhamento de fatores capazes de causar impactos negativos nas atividades organizacionais a fim de mitigá-lo (Senna et al., 2020b; Aghajanian, 2018).

\begin{tabular}{|l|l|l|l|l|l|}
\hline Rev. Gest. Soc. Ambient. & São Paulo (SP) & v.15 & p.01-18 & e02733 & 2021. \\
\hline
\end{tabular} 
No que tange a identificação, sua importância se dá na tentativa de entender já que ao compreender os riscos de fornecimento pode permitir que as organizações na sua atividade de compras tomem medidas eficazes em resposta a esses riscos. O seu objetivo é identificar infortúnios futuros para gerenciar esses cenários de forma proativa. Após a identificação é realizada a análise ou avaliação visando a priorização de riscos as quais são necessários para selecionar uma gestão adequada das ações para mitigação dos fatores de risco já identificados e reconhecidos. Por fim é realizado o controle ou acompanhamento que é parte da estratégia da mitigação de risco a qual tem sido uma das etapas de gerenciamento de risco comumente usadas (Wibowo \& Sundermeier, 2020; Aghajanian, 2018; Chan et al., 2011).

Numa alusão às práticas de compras públicas sustentáveis, destaca-se que os riscos de incompatibilidade entre a oferta e a demanda, os riscos de custos de estoque mais elevados se apresentam como um fator relevante na decisão de compra (Zepeda et al., 2016). Nesse contexto, a gestão logística como a parte responsável por controlar, planejar e implementar o fluxo desde o ponto de origem até o ponto de consumo também deve atentar aos riscos que afetam a cadeia de suprimentos públicos, uma vez que, ao se tratar de uma organização pública, os desafios se revelam ainda maiores tendo em vista a importância da manutenção do funcionamento das atividades estatais. Assim, tratar o risco como a possibilidade daquilo que é esperado não se confirmar, não implica necessariamente em algo indesejável, mas em não confirmação, tão somente, do que foi vislumbrado (Senna et al., 2020b; Imran et al., 2018; Oliveira \& Musetti, 2014; Chen et al., 2013).

\subsection{Sustentabilidade em compras públicas}

As compras públicas sustentáveis (CPS) têm ganhado relevância a cada dia em face da capacidade de influência que o estado detém para modificar e criar mercados. No cumprimento dos Objetivos de Desenvolvimento Sustentáveis (ODS) preconizado pela Organização das Nações Unidas (ONU), por meio da Agenda 2030, as compras públicas sustentáveis são importantes estímulos ao consumo e a produção responsáveis. E para isso, os países em desenvolvimento e desenvolvidos estão gastando bilhões de dólares para atingi-los. Contudo, pouca atenção é dada às compras públicas como um impulsionador do desenvolvimento sustentável (Chowdhury et al., 2019; Gallon et al., 2019; Snider et al., 2013; Zhu et al., 2013).

As CPS referem-se a compras de bens e serviços os quais buscam a relação custo-benefício atrelados às preocupações ambientais, econômicas e sociais (Waris et al., 2019; Shafiq et al., 2017; Ruparathna \& Hewage, 2015; Elkington, 1998). Uma vez que a aquisição sustentável pode ser identificada como uma prioridade junto à conformidade com aquisições regulares (Roman, 2017; Walker, 2015; Walker \& Brammer, 2012; Walker \& Brammer, 2009). Walker e Brammer (2009) apontam que as compras sustentáveis é a atividade que seguem os princípios do desenvolvimento sustentável, buscando garantir uma sociedade forte, saudável e justa, vivendo dentro dos limites ambientais e que promova a boa governança.

Caracterizada como o processo de integração dos aspectos ambientais, sociais, econômicos nas aquisições e contratações realizadas pelo poder público, as CPS referem-se a compras de bens e serviços as quais buscam a relação custo-benefício atrelada às preocupações ambientais, econômicas e sociais, uma vez que a aquisição sustentável pode ser identificada como uma prioridade junto à conformidade com aquisições públicas regulares (Waris et al., 2019; Shafiq et al., 2017; Ruparathna \& Hewage, 2015; Tvaronavičiene, 2012; Elkington, 1998). 
Diante disso, a gerenciamento de riscos em compras públicas deve se estender também à adoção de critérios de sustentabilidade nos procedimentos de seleção e na habilitação de fornecedores às necessidades do estado os quais culminam em compras públicas. Assim, o gerenciamento de riscos em compras públicas sustentáveis (Grcps) deve observar necessariamente em suas etapas questões ambientais, econômicas e sociais, bem como os tipos de riscos decorrentes dessa adoção, tais como não entrega do material, falta de forneced ores em conformidades com as práticas sustentáveis, desastres naturais, e outros (Gallon et al., 2019; Aghajanian, 2018; Mysiak \& Dionisio PérezBlanco, 2016).

Nesse sentido, as compras públicas, tais como prática contemporânea de estímulos ao desenvolvimento de uma sociedade sustentável, se consolidarão por meio da mitigação dos riscos e adoção dessa prática como elemento norteador da aquisição pública.

\section{METODOLOGIA}

Esta pesquisa parte do anseio em identificar, na literatura contemporânea, o estado da arte por meio dos autores, trabalhos e periódicos mais relevantes sobre o tema gerenciamento de riscos inerentes às compras públicas que tenham um viés sustentável como critério no processo de aquisição, tendo a seguinte pergunta norteadora: Quais são os autores, artigos e periódicos mais relevantes sobre o gerenciamento de riscos em compras públicas sustentáveis na última década?

Para responder à questão de pesquisa, realizou-se a análise bibliométrica a partir do levantamento bibliográfico de artigos em inglês nas bases científicas Scopus e Web of Science na temática de gerenciamento de riscos e compras públicas sustentáveis entre os anos de 2010 a 2020. Roesch (2013) destaca que a busca literária permite levantar soluções alternativas para tratar de uma problemática, o que possibilita conhecer quadros de referência alternativos, investindo na consciência crítica do pesquisador. Segundo Derviş (2019), métodos bibliométricos são usados para avaliar a produtividade dos resultados científicos quantitativamente. Nesse sentido, a análise bibliométrica buscou identificar quanto ao número de publicações por ano, número de publicações e citações por país, os dez principais autores e países na temática pesquisada, número de citações por artigo, número de publicação por periódico, co-citação e colaboração, bem como da convergência temática das palavras-chave das pesquisas realizadas.

Assim, inicialmente, para o desenvolvimento da análise bibliométrica utilizou-se um grupo de palavras-chave que orbita à temática do trabalho para a busca nas bases científicas Scopus e Web of Science. Sendo assim, utilizando-se o seguinte conjunto de descritores no idioma inglês: ("Risk Management" OR "Risk Assessment ") AND (("Public Procurement" OR "Government Procurement") AND ("Sustainable" OR "Sustainability" OR "Green")), a qual buscou a convergência temática entre o gerenciamento de riscos (GR) e as compras públicas sustentáveis (CPS).

Uma vez realizada as buscas nas bases indicadas, foram baixados os arquivos em formato . bib correspondentes para realização da análise no Software $R$ Studio, utilizando a seguinte linha de comando para extração e consolidação dos dados no arquivo pesquisa.RData para análise no Biblioshiny, disposto na Figura 1: 


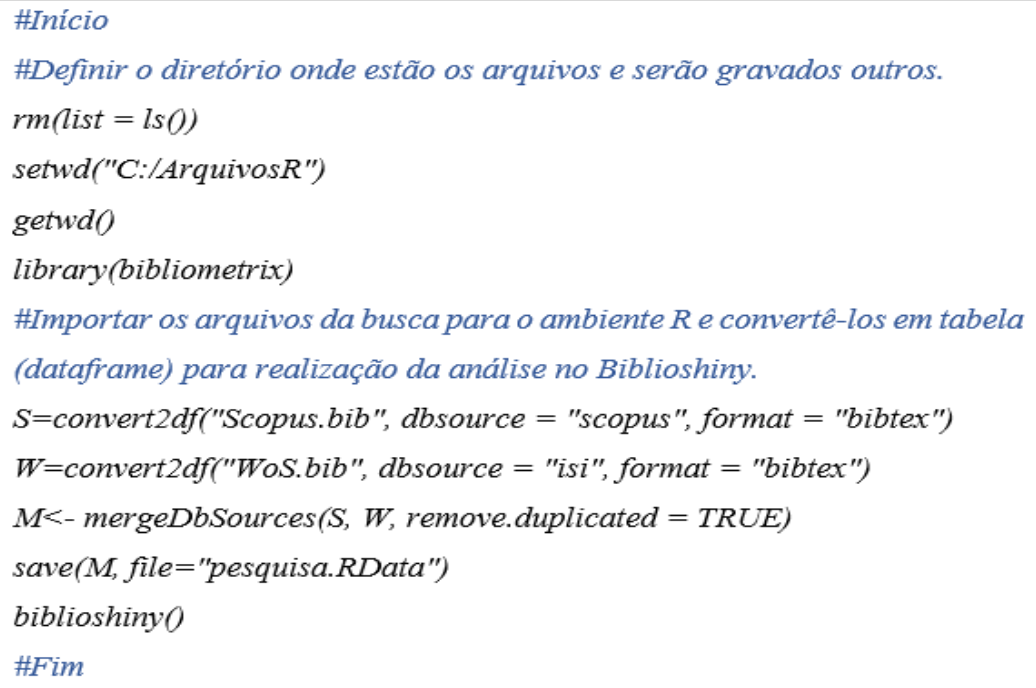

Figura 1: Linha de comando para extração de dados no Software R Studio Fonte: Elaborado pelos autores

O Biblioshiny é uma plataforma de análise bibliométrica a partir de dados coletados nas bases científicas cuja interface gráfica é baseada na web, por meio de arquivos consolidados na linguagem $\mathrm{R}$, a qual se destaca ao incorporar uma grande variedade de análises (Aria \& Cuccurullo, 2017). A linguagem R, criada pelos pesquisadores Brian Ripley e Bill Venables, é uma linguagem de programação de código aberto e gratuito que mais cresce em uso na atualidade (Ferreira, 2020; MoralMuñoz et al., 2020).

A Figura 2 apresenta o resumo o fluxo da metodologia de pesquisa utilizada no levantamento bibliográfico realizad o nas bases científicas.

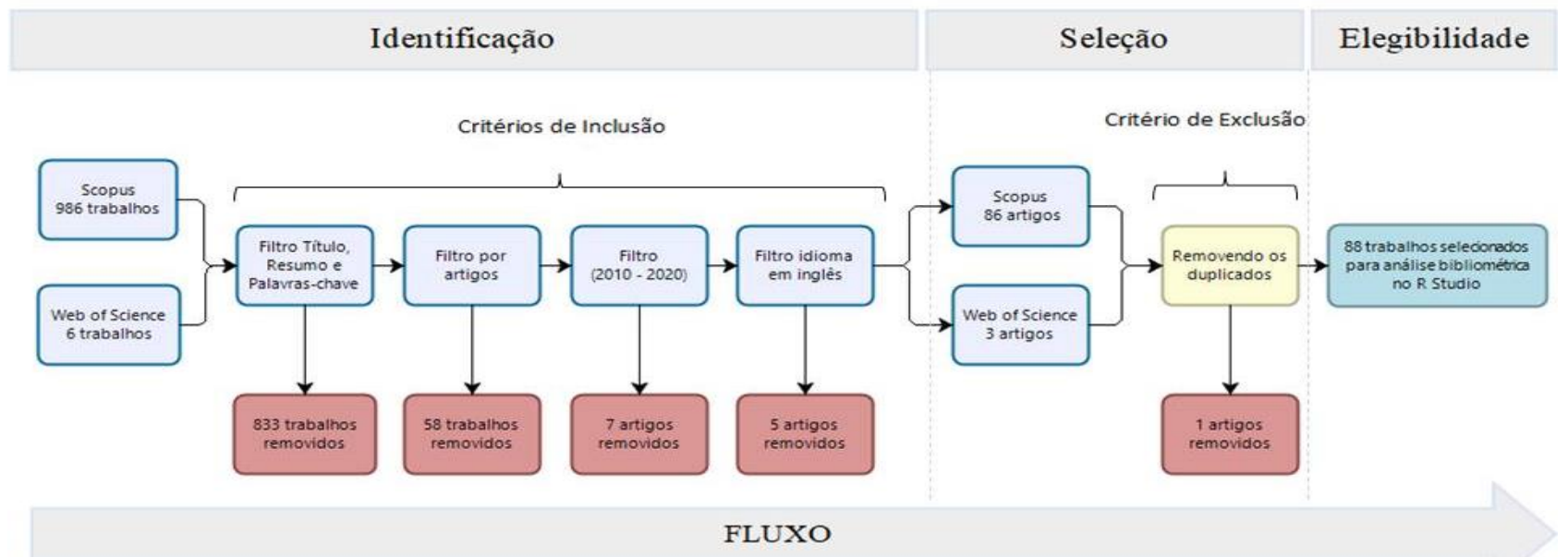

Figura 2: Fluxo da busca nas bases seleciona das para análise bibliométrica

Fonte: Elaborado pelos autores

$\mathrm{Na}$ etapa de identificação, foi realizada para a coleta a aplicação do critério de inclusão com filtros pelo título, resumo e palavras-chave, artigos, anos e trabalhos apenas no idioma inglês. Na segunda etapa, a Seleção foi aplicada remoção de trabalhos duplicados como critério de exclusão. E, por fim, a etapa de Elegibilidade, a qual cuidou de resumir os trabalhos selecionados para compor a análise bibliométrica. Assim, dos 89 artigos indicados a partir dos descritores propostos, um estava em duplicidade em ambas às bases, sendo excluído da próxima etapa de análise, restando assim 88 artigos para compor a análise bibliométrica, por meio do software $R$ Studio, para validação da convergência do campo temático dos trabalhos selecionados no Biblioshiny. 


\section{RESULTADOS BIBLIOMÉTRICOS}

Com a evolução dos sistemas de informações, os processos de recuperação de dados também foram aprimorados, como os sistemas de indexação de periódicos, livros, teses, etc. os quais facilitam o processo de busca bibliográfica e análises estatísticas da evolução científica do tema pesquisado em determinada área do conhecimento (Lacerda et al., 2012). O conceito de análise bibliométrica se baseia em parâmetros observáveis de um conjunto de artigos selecionados a partir de uma evidenciação quantitativa dos parâmetros definidos conforme intenção de pesquisa, se colocando como uma ferramenta estatística que permite não somente mapear, mas também gerar diferentes indicadores do conhecimento (Lacerda et al., 2012; Guedes \& Borschiver, 2005). Vanti (2002) nos ensina que, a partir da bibliometria, é possível definir uma lista de publicações e periódicos como evidência científica, além de prever as demandas de pesquisas futuras por meio da evolução histórica de publicações.

Assim, nesta seção, destacam-se os resultados numéricos da análise bibliométrica a partir da consulta às bases selecionadas. Com isso, observa-se que essa etapa contempla os trabalhos extraídos das bases em que a Figura 3 mostra a distribuição dos artigos entre 2010 e 2020, num levantamento realizado no primeiro trimestre de 2021, somando ao todo 88 artigos publicados.

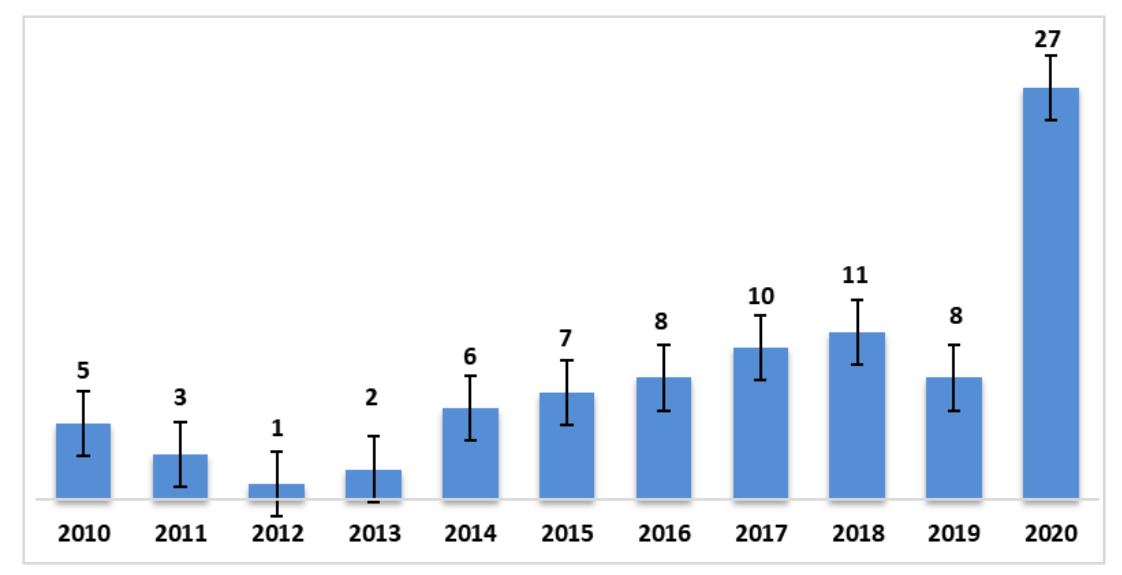

Figura 3: Distribuição de artigos por ano

Fonte: Elaborado pelos autores

O que se observa da Figura 3 é um crescimento tímido no número de publicações sobre riscos em compras públicas sustentáveis a partir de 2014. Contudo, em 2020 parece que a temática se confirma como campo de interesse de pesquisa da academia, uma vez que se tem um aumento de quase três vezes o número de publicação no ano anterior. Em 2010, foram publicados cinco documentos e dez anos depois são registrados 27 , o que representa uma taxa de crescimento de $540 \%$ entre o ano base 2010 e 2020.

A produção por países também é um aspecto interessante a ser observado pela análise bibliométrica realizada. A Figura 4 destaca os dez principais países geradores de pesquisa nessa temática. 


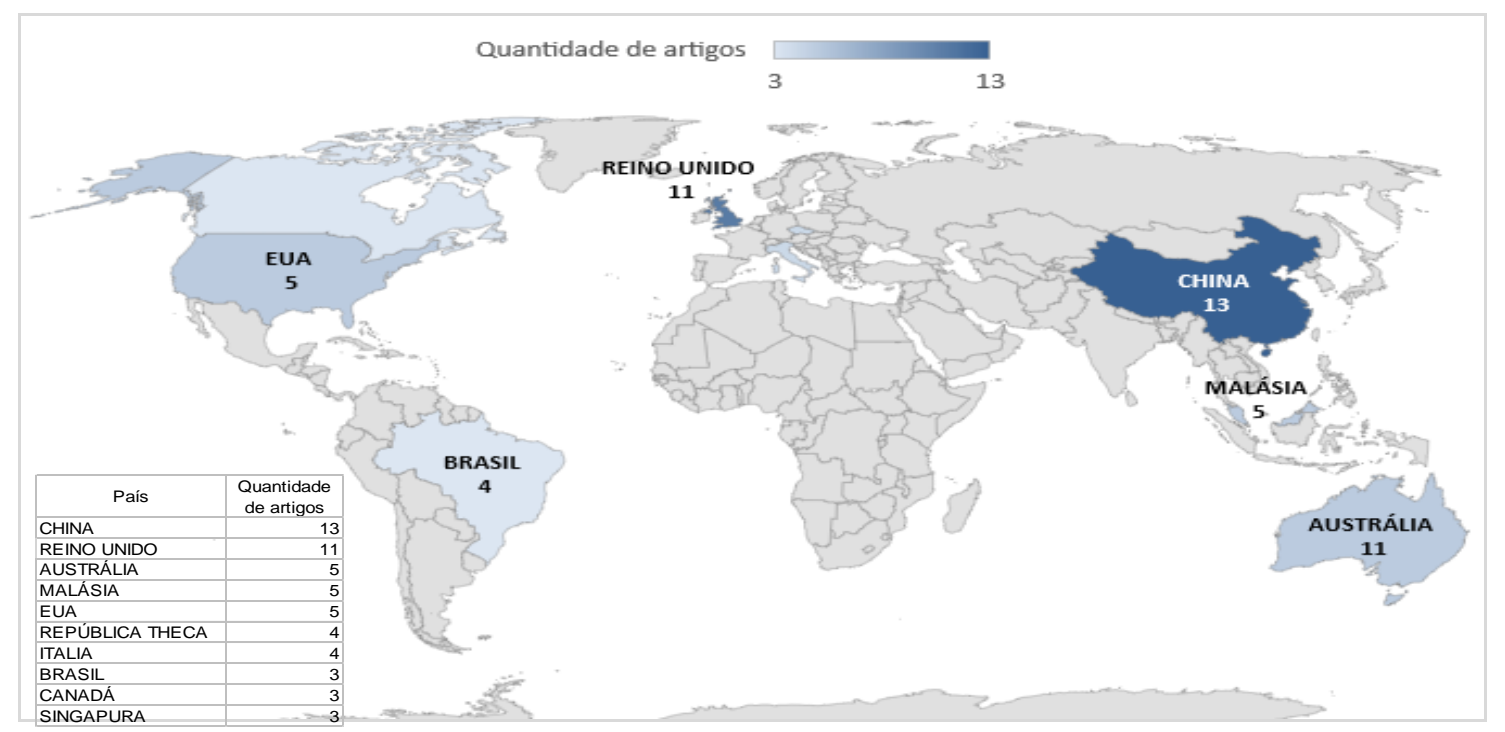

Figura 4: Os dez principais países publicadores

Fonte: Elaborado pelos autores

Na Figura 4, a China lidera em número de publicações no período levantado sobre a temática das compras públicas sustentáveis e sua relação com o gerenciamento de riscos, representando $23 \%(\mathrm{n}=13)$ do total de artigos publicados, seguida pelo Reino Unido com 19,30\% ( $\mathrm{n}=11)$, em que esses dois países contribuíram com mais da metade das produções, confirmando que o poder de compras públicas pode ser um importante impulsionador para compras ecologicamente corretas (Cheng et al., 2018). O Brasil figura na oitava posição com três trabalhos publicados no período de análise. Assim, trabalhar com conceito de sustentabilidade em compras públicas atrelada à gestão de riscos parece ser importante e relevante para muitos países diferentes.

Além disso, quando se trata de número de citações (Figura 5), Hong Kong é o país que lidera nesse quesito, representando $34 \%(n=326)$ dos dez países mais citados, seguido pelo Reino Unido com 22,65\% ( $\mathrm{n}=214)$.

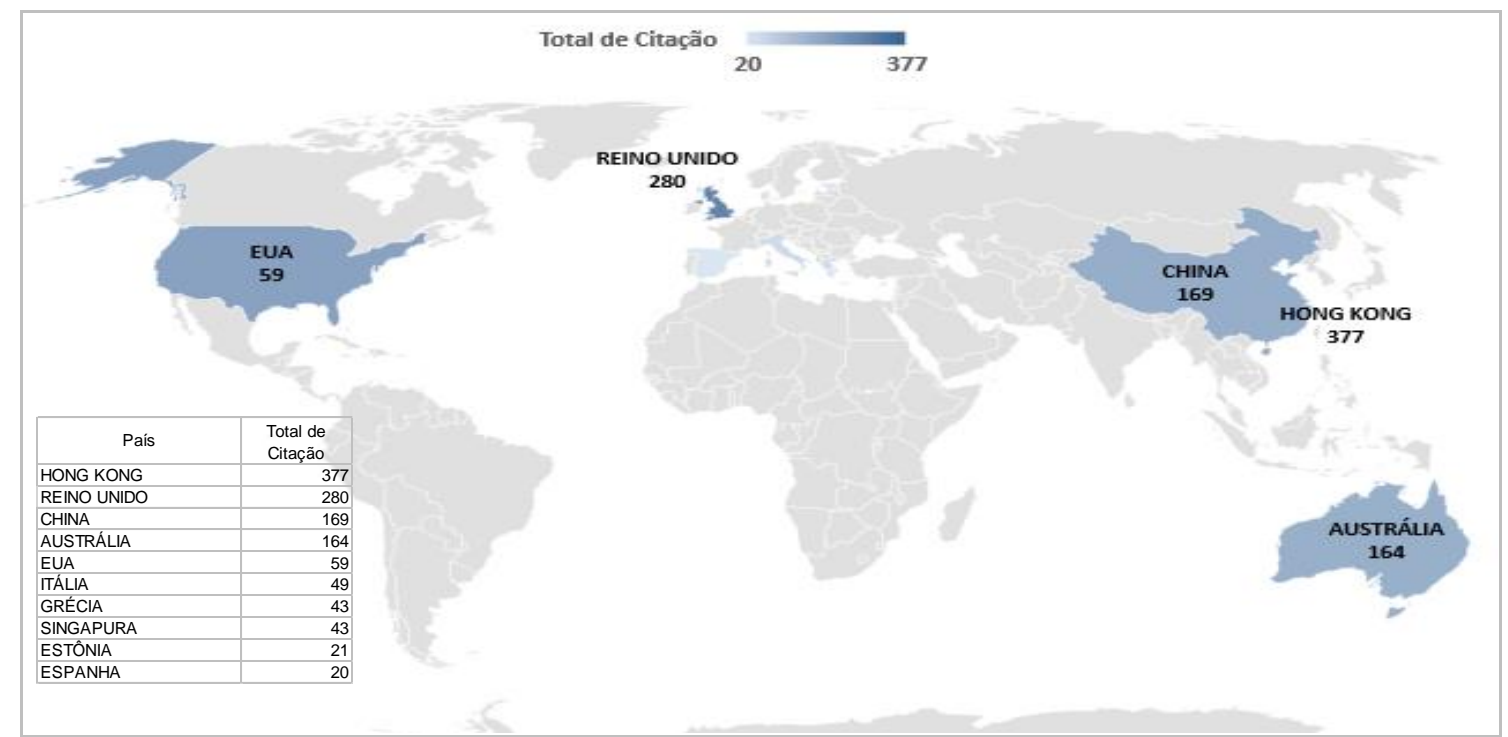

Figura 5: Os dez principais países com mais citações Fonte: Elaborado pelos autores 
A relevância autoral, em via de regras é medida pela quantidade de trabalhos que determinado autor publica frente a determinado campo do conhecimento, assim como o número de citações que os trabalhos desse autor consegue angariar. Nesse sentido, a área de conhecimento dos quais os trabalhos analisados estão inseridos refletem o interesse nesse campo de pesquisa. Para todas as publicações analisadas, os assuntos mais comuns são Negócios, Gestão e Contabilidade, Engenharia e Ciências Sociais $(22,65 \%, 20,44 \%$ e $17,13 \%)$ dentre os 14 assuntos, conforme Tabela 1.

Tabela 1: Participação na área de conhecimento.

\begin{tabular}{|c|c|}
\hline \multicolumn{2}{|l|}{1 apela 1: Participaçao na area de } \\
\hline Área de conhecimento & Participação \\
\hline Negócios, gestão e contabilidade & $22,65 \%$ \\
\hline Engenharia & $20,44 \%$ \\
\hline Ciências sociais & $17,13 \%$ \\
\hline Ciência ambiental & $11,05 \%$ \\
\hline Energia & $6,63 \%$ \\
\hline Economia, econometria e finanças & $5,52 \%$ \\
\hline Medicina & $4,42 \%$ \\
\hline Ciências da decisão & $3,87 \%$ \\
\hline Ciência da computação & $3,31 \%$ \\
\hline Matemática & $2,21 \%$ \\
\hline Enfermagem & $1,10 \%$ \\
\hline Artes e humanidades & $0,55 \%$ \\
\hline Ciências da terra e planetárias & $0,55 \%$ \\
\hline Psicologia & $0,55 \%$ \\
\hline
\end{tabular}

Fonte: Elaborado pelos autores

As áreas de conhecimentos em Negócios, Gestão e Contabilidade, Engenharia e Ciências Sociais são as categorias mais exploradas por estudos no campo temático investigado, podendo-se concluir que há espaço proeminente para áreas como a Ciência Ambiental e Economia, as quais têm muito a contribuir com evidências por meio de pesquisas se utilizand o da metodologia de estudos de casos como estratégia de pesquisa.

Já na Figura 6, são apresentados os autores mais relevantes nesse campo de pesquisa em número de publicações.

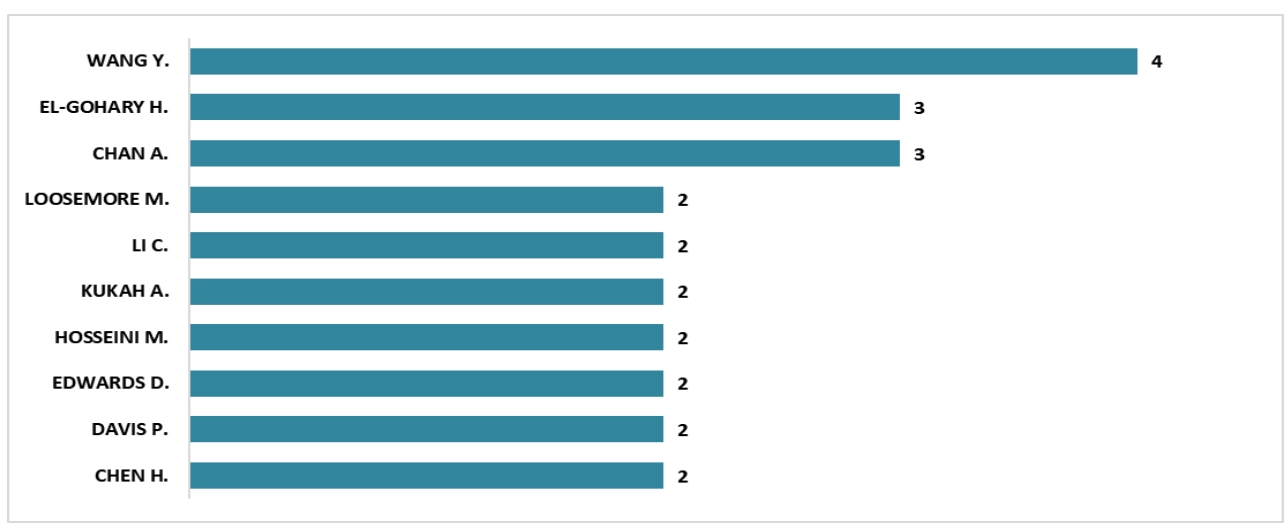

Figura 6: Autores mais produtivos.

Fonte: Elaborado pelos autores. 
Juntos, os autores Shou Wang, Albert Chan e Hatem El-Gohary, que possuem dez publicações sobre o tema, são os que mais publicaram. Albert Chan é professor e chefe associado, do Departamento de Construção e Imobiliário da Universidade politécnica em Hong Kong. Shou Wang é professor do Departamento de Gerenciamento de Construção na Universidade de Tsinghua, em Pequim na China. Já Hatem El-Gohary é professor e editor-chefe do International Journal of Online Marketing e editor associado do The International Journal of Customer Relationship Marketing and Management.

Os títulos dos dez artigos mais citados a partir da análise bibliométrica apontados pelo Biblioshiny realizada estão dispostos na Tabela 2:

Tabela 2: Título dos dez artigos mais citados

\begin{tabular}{|c|c|c|}
\hline Título & Autores & $\begin{array}{l}\text { Total de } \\
\text { citações }\end{array}$ \\
\hline $\begin{array}{l}\text { Are public-private partnerships a healthyoption? A systematic } \\
\text { literature review }\end{array}$ & (Roehrich et al., 2014) & 173 \\
\hline $\begin{array}{l}\text { Empirical Study of Risk Assessment and Allocation of Public- } \\
\text { Private Partnership Projects in China }\end{array}$ & (Chan et al., 2011) & 155 \\
\hline $\begin{array}{l}\text { Schedule risks in prefabrication housing production in Hong } \\
\text { Kong: a social network analysis }\end{array}$ & (Li et al., 2016) & 94 \\
\hline $\begin{array}{l}\text { Conceptual Frameworkfor the Performance Measurement of } \\
\text { Public-Private Partnerships }\end{array}$ & (J. Liu et al., 2015) & 72 \\
\hline $\begin{array}{l}\text { Toward the betterment of risk allocation: Investigating risk } \\
\text { perceptions of Australian stakeholder groups to public-private- } \\
\text { partnership tollroad projects }\end{array}$ & (Chung et al., 2010) & 58 \\
\hline Risks in Energy Performance Contracting (EPC) projects & (Lee et al., 2015) & 50 \\
\hline $\begin{array}{l}\text { First Public-Private-Partnership Application in Taiwan's } \\
\text { Wastewater Treatment Sector: Case Study of the Nanzih BOT } \\
\text { Wastewater Treatment Project }\end{array}$ & (Zheng \& Tiong, 2010) & 38 \\
\hline $\begin{array}{l}\text { Public-private partnerships and investments in innovation: the } \\
\text { influence of the contractual arrangement }\end{array}$ & $\begin{array}{l}\text { (Roumboutsos \& Saussier, } \\
\text { 2014) }\end{array}$ & 34 \\
\hline $\begin{array}{l}\text { Cross-efficiency evaluation in data envelopment analysis based } \\
\text { on prospect theory }\end{array}$ & (H.-H. Liu et al., 2019) & 33 \\
\hline $\begin{array}{l}\text { Public-Private Integrated Partnerships Demonstrate The } \\
\text { Potential To Improve Health Care Access, Quality, And } \\
\text { Efficiency }\end{array}$ & (Sekhri et al., 2011) & 31 \\
\hline
\end{tabular}

Fonte: Elabora do pelos autores

$\mathrm{O}$ artigo mais citado dentre os dez, Are public-private partnerships a healthy option? A systematic literature review é um trabalho publicado em 2014, cuja proposta de pesquisa foi realizar a revisão sistemática da literatura a qual analisou mais de 1.400 publicações de uma ampla gama de disciplinas ao longo de um período de 20 anos indexados à base Web of Science.

Já os periódicos mais relevantes quando se trata de gerenciamento de riscos em compras públicas sustentáveis estão dispostos na Tabela 3, logo abaixo: 
Santos, A. L. T. dos, \& Reis, A. da C. (2021). Tendências Teóricas do Gerenciamento de Riscos em Compras Públicas Sustentá veis: Uma Análise Bibliométrica A Partir das Bases Scopus e Web of Science.

Tabela 3: Periódicos mais relevantes

\begin{tabular}{lcc}
\hline Nome dos periódicos & $\begin{array}{c}\text { Quantidade } \\
\text { de artigos }\end{array}$ & $\begin{array}{c}\text { Fator de Impacto: } \\
\text { Índice H }\end{array}$ \\
\hline Journal of Cleaner Production & 5 & 5 \\
\hline Sustainability (Switzerland) & 4 & 2 \\
\hline Journal of Engineering Design and Technology & 4 & 3 \\
\hline Management and Marketing & 3 & 1 \\
\hline Construction Management and Economics & 2 & 2 \\
\hline Facilities & 2 & 2 \\
\hline Journal of Construction Engineering and Management & 2 & 1 \\
\hline Public Money and Management & 2 & 2 \\
\hline Amfiteatru Economic & 1 & 1 \\
\hline Annali di Igiene Medicina Preventiva e di Comunita & 1 & 1 \\
\hline
\end{tabular}

Fonte: Elaborado pelos autores

O Journal of Cleaner Production é um periódico de publicação acadêmica revisada por pares que cobre pesquisas transdisciplinares sobre produção limpa. É o período com maior número de publicações no período com um total de cinco publicações. Seguido pelo periódico Sustainability com quatro publicações. A Sustainability é uma revista internacional, interdisciplinar, acadêmica, revisada por pares e de acesso aberto, sobre sustentabilidade ambiental, cultural, econômica e social, oferecendo um fórum avançado para estudos relacionados à sustentabilidade e ao desenvolvimento sustentável.

Ademais, buscou-se conhecer os termos mais fortes no campo temático proposto para a presente pesquisa, sendo risk management revelando-se o verbete mais evidente dentre os demais, como se observa na Figura 7.

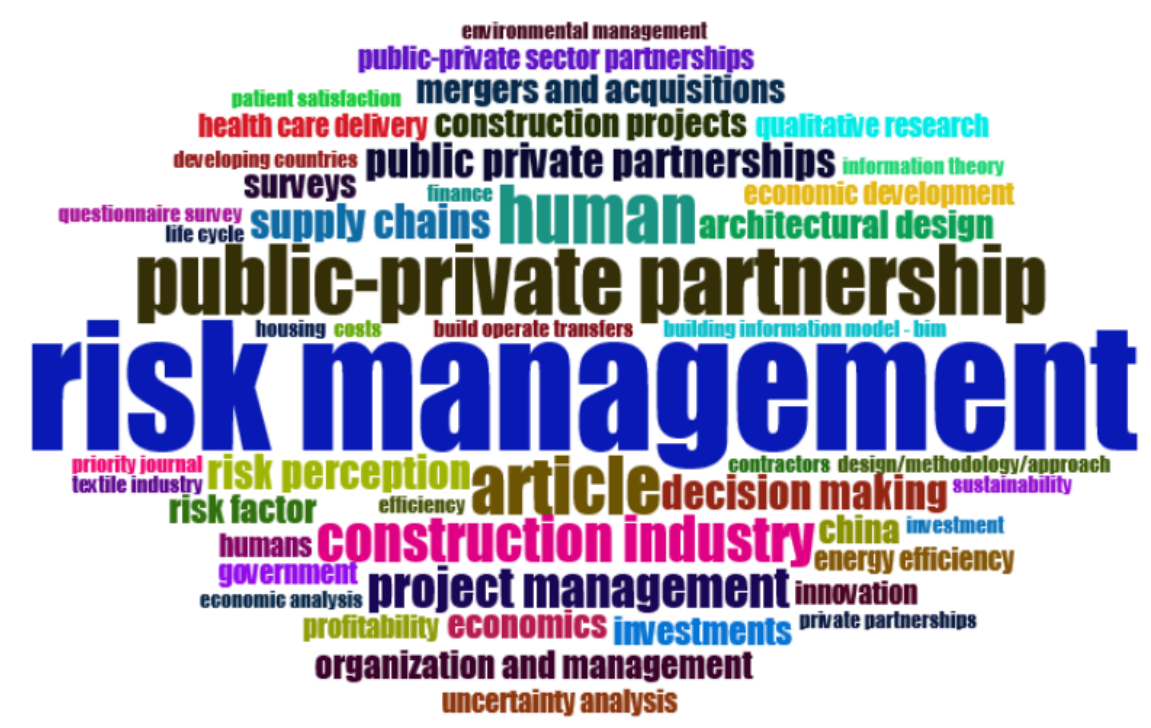

Figura 7: Nuvem de palavras

Fonte: Elaborado pelos autores

Geralmente, riscos estão atrelados às oportunidades a que o negócio vislumbra em seu segmento de atuação, já que o risco pode ser entendido como a probabilidade ou possibilidade de um infortúnio ou insucesso, assim como um resultado indesejado em face de um objetivo almejado (Kalvet \& Lember, 2010; Alexandre et al., 2004). Observa-se, a partir dos termos mais recorrentes, que a palavra sustainability ainda é um tema periférico quando se trata de correlacioná-lo com o risk management em compras 
públicas. O que sugere que essa linha de pesquisa ainda é incipiente e confirma um longo caminho nas consolidações de ações de sustentabilidade estar associadas às práticas de gerenciamento de riscos, pois observar a perspectiva de um grupo de palavras-chave implica mudar a forma como se pensa sobre os conceitos a elas atrelados. A análise de palavras é uma ferramenta poderosa para análise de conteúdo e pode ser usada para prever o potencial de crescimento futuro e direção do conhecimento (Bardin, 2016).

Na Figura 8 é apresentada uma rede de co-citações, ou seja, artigos que citam uns aos outros, tendo alguns autores como principais destacados na imagem da rede, em que na rede de colaboração científica destacam-se quatro crusters principais. A suposição básica, por trás da análise de co-citação, é destacar o documento relevante a partir do trabalho bem-sucedido realizado na área de assunto (Feng et al., 2015; Tsay et al., 2003).

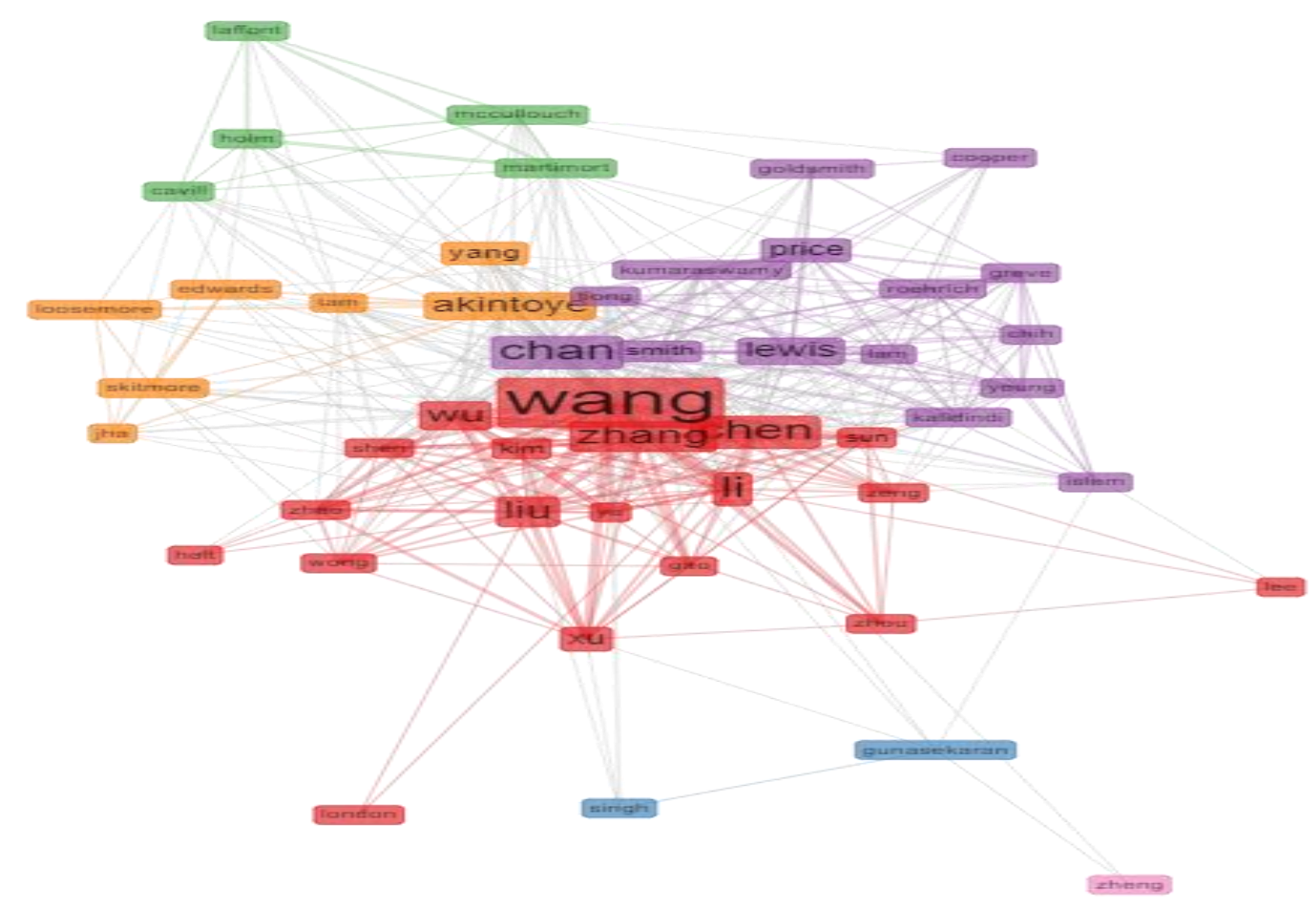

Figura 8: Rede de cocitações

Fonte: Elaborado pelos autores

Os clusters são formados de acordo com as semelhanças das referências citadas por artigos publicados (Feng et al., 2015). Assim, na Figura 8, a separação de quatro clusters, identificados por cor, em que cada um destes clusters tem artigos principais destacados com maior tamanho de fonte ante de quanto maior a quantidade de citações for pelos outros autores.

Diante disso, pode-se verificar a evolução de pesquisas sobre gerenciamento de riscos em compras públicas sustentáveis, mesmo que incipientes, analisando e constatando autores, revistas e artigos relevantes sobre o tema. Fica demonstrado que ainda existe um longo caminho a ser percorrido para que tal temática se consolide na literatura. 


\section{CONSIDERAÇÕES FINAIS}

Assim como o gerenciamento de risco da cadeia de suprimentos (GRCS) cresceu em importância, o gerenciamento de riscos em compras públicas tem se tornado uma das tendências gerenciais mais importantes da atualidade, carecendo, contudo, de mais estudos sobre seus impactos em compras públicas que aplicam critérios de sustentabilidade nas etapas de contratação. A fim de contribuir para os estudos nessa temática, este trabalho teve por objetivo apontar tendências teóricas a partir de um estudo bibliométrico dos principais trabalhos sobre o gerenciamento de riscos em compras públicas sustentáveis. Para isso, revelou-se por meio de análise bibliométrica o número de publicações por ano, quantidade de citações por autor e países que mais tratam dessa temática, além de identificar os autores mais relevantes no tema pesquisado, bem como da convergência temática das palavras-chave das pesquisas realizadas. O levantamento bibliográfico realizado nas bases científicas Scopus e Web of Science contemplando os anos de 2010 a 2020, identificando 89 trabalhos em inglês para análise bibliométrica por meio do pacote bibliometrix do Software R Studio.

Diante disso, este estudo revela que as pesquisas que envolvam os campos temáticos da gestão de riscos (GR) e compras públicas sustentáveis (CPS) se mostram uma tendência teórica ainda em crescimento, uma vez que, a partir do levantamento bibliométrico do volume de publicação na última década (2010-2020), se revela um crescimento de cinco vezes no número de trabalhos publicados entre o primeiro e o último ano de coleta. Todavia, a partir da análise da nuvem de palavras, é possível inferir que o termo risk management ainda é o termo central ou base para a ampliação das discussões sobre as práticas sustentáveis como fator de riscos em compras públicas. Além disso, as áreas de conhecimentos em Negócios, Gestão e Contabilidade, Engenharia e Ciências Sociais são as categorias mais exploradas por estudos no campo temático investigado, concluindo-se que há espaço proeminente para as áreas como a Ciência Ambiental e Economia, as quais têm muito a contribuir com evidências por meio de pesquisas empíricas se utilizando da metodologia de estudos de casos como estratégia de pesquisa.

Por meio da análise realizada pela plataforma de análise bibliométrica Biblioshiny foi possível identificar o trabalho Are public-private partnerships a healthy option? A systematic literature review dos autores Roehrich, J. K., Lewis, M. A. e George, G. como o mais relevante em face de seu número de citações $(n=173)$, publicado no periódico Social Science and Medicine no ano de 2014. Além disso, análise revelou também que o Journal of Cleaner Production foi o periódico que mais publicou sobre a temática investigada $(n=5)$. Os autores Shou Wang, Albert Chan e Hatem El-Gohary foram os que mais publicaram entre 2010 e 2020, possuindo dez publicações sobre a temática. Quantos aos países que mais produziram, cabe destaque para a China com 13 trabalhos, seguida pelo Reino Unido com 11. Todavia, em matéria de número de citações Hong Kong ( $\mathrm{n}=326)$ supera países como Reino Unido $(\mathrm{n}=214)$, Austrália (n=134) e China (n=81), representando 34\% do total de citações dos países.

Por fim, para se investigar de forma empírica o gerenciamento de riscos em compras públicas sustentáveis como prática contemporânea de estímulos ao desenvolvimento de uma sociedade cada vez mais sustentável, novos estudos merecem atenção pelos pesquisadores do tema. Nesse contexto, avaliar qual a influência da administração pública no gerenciamento de riscos em cadeia de suprimentos sustentáveis a partir do seu poder de compra também tem seu espaço numa proposta de estudo futuro. Ou seja, sugere-se que uma pesquisa futura poderá analisar qualitativamente no portfólio bibliográfico internacional, com a finalidade de se revisar 
sistematicamente quais categorias de riscos mais afetam a cadeia de suprimentos em razão da adoção de critérios de sustentabilidade nas etapas do processo de compras públicas. Ademais, cabe identificar, a partir de revisão integrativa da literatura, quais as estratégias de mitigação de riscos inerentes são mais adequadas ao se estabelecer critérios de sustentabilidade nos procedimentos de compras e contratações de serviços, aos desafios de uma compra pública sustentável eficiente por parte do estado.

\section{REFERÊNCIAS}

Aghajanian, S. (2018). An empirical procurement risk management framework in supply chain networks: A hybrid approach. Industrial Engineering and Management Systems, 17(4), 730-744.

Aguas, J. P. Z., Adarme, W. A., \& Serna, M. D. A. (2013). Supply risk analysis: applying system dynamics to the Colombian healthcare sector. Ingenieria $e$ Investigacion, 33(3), 76-81.

Alexandre, F., Gambôa, R., \& Filho, E. B. (2004). Risk management method to erp systems implementation based on. Revista de Gestão Da Tecnologia e Sistemas de Informação, 1(1), 45-62.

Aria, M., \& Cuccurullo, C. (2017). bibliometrix: An R-tool for comprehensive science mapping analysis. Journal of Informetrics, 11(4), 959-975.

Bakir, S., Khan, S., Ahsan, K., \& Rahman, S. (2018). Exploring the critical determinants of environmentally oriented public procurement using the Dematel method. Journal of Environmental Management, 225, 325-335.

Bardin, L. (2016). Análise de conteúdo $\left(1^{\mathrm{a}}\right)$. Edições 70.

Brammer, S., \& Walker, H. (2011). Sustainable procurement in the public sector: An international comparative study. International Journal of Operations and Production Management, 31(4), 452-476.

Chan, A. P. C., Yeung, J. F. Y., Yu, C. C. P., Wang, S. Q., \& Ke, Y. (2011). Empirical study of risk assessment and allocation of public-private partnership projects in China. Journal of Management in Engineering, 27(3), 136-148.

Chen, D. Q., Preston, D. S., \& Xia, W. (2013). Enhancing hospital supply chain performance: A relational view and empirical test. Journal of Operations Management, 31(6), 391-408.

Cheng, W., Appolloni, A., D’Amato, A., \& Zhu, Q. (2018). Green public procurement, missing concepts and future trends - A critical review. Journal of Cleaner Production, $176,770-784$.

Chiarini, A., Opoku, A., \& Vagnoni, E. (2017). Public healthcare practices and criteria for a sustainable procurement: A comparative study between UK and Italy. Journal of Cleaner Production, 162, 391-399.

Chowdhury, N. A., Ali, S. M., Mahtab, Z., Rahman, T., Kabir, G., \& Paul, S. K. (2019). A structural model for investigating the driving and dependence power of supply chain risks in the readymade garment industry. Journal of Retailing and Consumer Services, $51,102-113$.

Chung, D., Hensher, D. A., \& Rose, J. M. (2010). Toward the betterment of risk allocation: Investigating risk perceptions of Australian stakeholder groups to public- 
private-partnership tollroad projects. Research in Transportation Economics, 30(1), 4358.

Cortis, N., Fang, Q., \& Dou, Z. (2018). Social service purchasing in China: Rationale, features, and risks. Asian Social Work and Policy Review, 12(3), 200-208.

Cruz, E. C., Olivera, T., \& Gurgel, A. M. (2020). Desenvolvimento de um framework para o planejamento de compras públicas: estudo em uma Universidade Federal. Revista Gestão e Projetos, 11(3), 94-116.

Derviş, H. (2019). Bibliometric analysis using bibliometrix an R Package. Journal of Scientometric Res, 8(3)

Eckerd, A., \& Girth, A. M. (2017). Designing the buyer-supplier contract for risk management: assessing complexity and mission criticality. Journal of Supply Chain Management, 53(3), 60-75.

Elkington, J. (1998). Partnerships from cannibals with forks: the triple bottom line of 21 st-century business. Environmental Quality Management, 8(1), 37-51.

Feng, F., Zhang, L., Du, Y., \& Wang, W. (2015). Visualization and quantitative study in bibliographic databases: A case in the field of university-industry cooperation. Journal of Informetrics, 9(1), 118-134.

Ferreira, E. B. (2020). O software R e a pesquisa com Alimentos e Nutrição. Revista Sustinere, 8(2), 611-616.

Gallon, I., Flores, G. M., Trevisan, M., \& Kneipp, J. M. (2019). Analysis of the sustainability criteria applied to biddings of a federal public university. Revista de Gestão Ambiental e Sustentabilidade, 8(2), 313-332.

Guedes, V. L., \& Borschiver, S. (2005). Bibliometria: uma ferramenta estatística para a gestão da informação e do conhecimento, em sistemas de informação, de comunicação e de avaliação científica e tecnológica. Encontro Nacional de Ciência Da Informação, $6(1), 1-18$.

Henrique, B. M., Sobreiro, V. A., \& Kimura, H. (2020). Contracting in Brazilian public administration: a machine learning approach. Expert Systems, 37(5).

Hyari, K. H. (2017). The Controversy around Unbalanced Bidding in Construction: Seeking a Fair Balance. Journal of Professional Issues in Engineering Education and Practice, 143(1).

Imran, M., Kang, C., \& Babar Ramzan, M. (2018). Medicine supply chain model for an integrated healthcare system with uncertain product complaints. Journal of Manufacturing Systems, 46, 13-28.

Ipea (2019). O mercado de compras governamentais brasileiro (2006-2017): mensuração e análise. Instituto de Pesquisa Econômica Aplicada - IIPEA.

Kalvet, T., \& Lember, V. (2010). Risk management in public procurement for innovation: The case of Nordic-Baltic Sea cities. Innovation, 23(3), 241-262.

Kashap, S. (2004). Public procurement as a social, economic and political policy. International Public Procurement Conference Proceedings, 3, 133-147.

Lacerda, R. T. O., Ensslin, L., \& Ensslin, S. R. (2012). Uma análise bibliométrica da literatura sobre estratégia e avaliação de desempenho. Gestão \& Produção, 19(1), 5978. 
Lee, P., Lam, P. T. I., \& Lee, W. L. (2015). Risks in energy performance contracting (EPC) projects. Energy and Buildings, 92, 116-127.

Li, C. Z., Hong, J., Xue, F., Shen, G. Q., Xu, X., \& Mok, M. K. (2016). Schedule risks in prefabrication housing production in Hong Kong: a social network analysis. Journal of Cleaner Production, 134, 482-494.

Liu, H.-H., Song, Y.-Y., \& Yang, G.-L. (2019). Cross-efficiency evaluation in data envelopment analysis based on prospect theory. European Journal of Operational Research, 273(1), 364-375.

Liu, J., Love, P. E. D., Davis, P. R., Smith, J., \& Regan, M. (2015). Conceptual framework for the performance measurement of public-private partnerships. Journal of Infrastructure Systems, 21(1).

Matos, A. L. T., Pires, S. R. I., Pizzinatto, N. K., \& Antoniolli, P. D. (2017). Panorama da Pesquisa Nacional sobre Gestão de Riscos em Cadeias de Suprimentos: Análise Bibliométrica no Período entre 2006 e 2015. Simpep, Bauru. Anais... Bauru: Simpósio de Engenharia de Produção 24, November.

Mehralian, G., Gatari, A. R., Morakabati, M., \& Vatanpour, H. (2012). Developing a suitable model for supplier selection based on supply chain risks: an empirical study from Iranian Pharmaceutical Companies. Iranian Journal of Pharmaceutical Research, 11(1), 209-219.

Mogre, R., Lindgreen, A., \& Hingley, M. (2017). Tracing the evolution of purchasing research: future trends and directions for purchasing practices. Journal of Business \& Industrial Marketing, 32(2), 251-257.

Moral-Muñoz, J. A., Herrera-Viedma, E., Santisteban-Espejo, A., \& Cobo, M. J. (2020). Software tools for conducting bibliometric analysis in science: An up-to-date review. Profesional de La Informacion, 29 (1), 1-20.

Mysiak, J., \& Dionisio Pérez-Blanco, C. (2016). Partnerships for disaster risk insurance in the EU. Natural Hazards and Earth System Sciences, 16(11), 2403-2419.

Norrman, A., \& Jansson, U. (2004). Ericsson's proactive supply chain risk management approach after a serious sub-supplier accident. International Journal of Physical Distribution and Logistics Management, 34(5), 434-456.

OCDE (2020). Valor gasto nos países da OCDE em compras públicas. Organização Para Economia Cooperação e Desenvolvimento - OCDE.

Oliveira, T. S., \& Musetti, M. A. (2014). Revisão compreensiva de logística hospitalar: conceitos e atividades. Revista de Gestão em Sistemas de Saúde, 03(01), 01-13.

Oliveira, U. R., Souza Espindola, L., Augusto, F., \& Marins, S. (2016). Analysis of supply chain risk management researches. Gest. Prod.

Roehrich, J. K., Lewis, M. A., \& George, G. (2014). Are public-private partnerships a healthy option? A systematic literature review. Social Science and Medicine, 113, 110119.

Roesch, S. M. A. (2013). Projetos de estágios e de pesquisa em Administração (Atlas (ed.); $3^{\text {a }}$.

Roman, A. V. (2017). Institutionalizing sustainability: a structural equation model of sustainable procurement in US public agencies. Journal of Cleaner Production, 143, 1048-1059.

\begin{tabular}{|l|l|l|l|l|l|l|}
\hline Rev. Gest. Soc. Ambient. & São Paulo (SP) & v.15 | & p.01-18 & e02733 & 2021. \\
\hline
\end{tabular} 
Roumboutsos, A., \& Saussier, S. (2014). Public-private partnerships and investments in innovation: the influence of the contractual arrangement. Construction Management and Economics, 32(4), 349-361.

Ruparathna, R., \& Hewage, K. (2015). Sustainable procurement in the Canadian construction industry: current practices, drivers and opportunities. Journal of Cleaner Production, 109(SI), 305-314.

Santos, L. R. L. dos. (2017). A fiscalização e o acompanhamento da execução do contrato administrativo na Administração Pública - artigo 67 - Lei No 8.666/93. Revista Digital Do Direito Administrativo, 4(2), 187-199.

Sekhri, N., Feachem, R., \& Ni, A. (2011). Public-private integrated partnerships demonstrate the potential to improve health care access, quality, and efficiency. Health Affairs, 30(8), 1498-1507.

Senna, P, Reis, A., Santos, I. L., Dias, A. C., \& Coelho, O. (2020). A systematic literature review on supply chain risk management: is healthcare management a forsaken research field? Benchmarking.

Senna, Pedro, Reis, A. da C., Sant'anna, D., \& Santos, A. C. de S. G. dos. (2020). Supply Chain Risk Management: a Bibliometric Analysis Considering Healthcare Supply Chains. Gestão de riscos em cadeias de suprimentos: uma análise bibliométrica considerando cadeias de suprimentos da saúde., 6(4), 1-15.

Shafiq, A., Johnson, P. F., Klassen, R. D., \& Awaysheh, A. (2017). Exploring the implications of supply risk on sustainability performance. International Journal of Operations and Production Management, 37(10), 1386-1407.

Snider, K. F., Halpern, B. H., Rend on, R. G., \& Kidalov, M. V. (2013). Corporate social responsibility and public procurement: how supplying government affects managerial orientations. Journal of Purchasing and Supply Management, 19(2), 63-72.

Tsay, M. Y., Xu, H., \& Wu, C. W. (2003). Author co-citation analysis of semiconductor literature. Scientometrics, 58(3), 529-545.

Tvaronavičiene, A. (2012). The possibilities to use public procurement as one of the instruments of implementation of sustainable development concept in republic of lithuania. Business: Theory and Practice, 13(3), 197-207.

Vanti, N. A. P. (2002). Da bibliometria à webometria: uma exploração conceitual dos mecanismos utilizados para medir o registro da informação e a difusão do conhecimento. Ciência da Informação, 31(2), 369-379.

Walker, H. (2015). New development: public procurement research at Ipsera - aligning research and practice, and future trends. Public Money and Management, 35(2), 141144.

Walker, H., \& Brammer, S. (2009). Sustainable procurement in the United Kingdom public sector. Supply Chain Management, 14(2),

Walker, H., \& Brammer, S. (2012). The relationship between sustainable procurement and e-procurement in the public sector. International Journal of Production Economics, 140(1), 256-268.

Waris, M., Panigrahi, S., Mengal, A., Soomro, M. I., Mirjat, N. H., Ullah, M., Azlan, Z. S., \& Khan, A. (2019). An Application of analytic hierarchy process (AHP) for sustainable procurement of construction equipment: multicriteria-based decision 
framework for Malaysia. Mathematical Problems in Engineering, 2019.

Wibowo, A., \& Sundermeier, M. (2020). Developing a fuzzy value-for-money analysis model for annuity-based public-private partnership infrastructure projects under consideration of risk mitigation capability. Journal of Construction Engineering and Management, 146(7).

Yazdani, M., Gonzalez, E. D. R. S., \& Chatterjee, P. (2019). A multi-criteria decisionmaking framework for agriculture supply chain risk management under a circular economy context. Management Decision.

Zaidi, S. A. H., Mirza, F. M., Hou, F., \& Ashraf, R. U. (2019). Addressing the sustainable development through sustainable procurement: What factors resist the implementation of sustainable procurement in Pakistan? Socio-Economic Planning Sciences, 68 .

Zepeda, E. D., Nyaga, G. N., \& Young, G. J. (2016). Supply chain risk management and hospital inventory: Effects of system affiliation. Journal of Operations Management, 44, 30-47.

Zheng, S., \& Tiong, R. L. K. (2010). First public-private-partnership application in Taiwan's wastewater treatment sector: case study of the Nanzih BOT wastewater treatment project. Journal of Construction Engineering and Management, 136(8), 913922.

Zhu, Q., Geng, Y., \& Sarkis, J. (2013). Motivating green public procurement in China: An individual level perspective. Journal of Environmental Management, 126, 85-95. 